\title{
Simvastatin Sensitizes
}

Radioresistant Prostate Cancer Cells by Compromising DNA Double-Strand Break Repair

\author{
Yu-An Chen', Hua-Wei Shih', Yi-Chun Lin', Hui-Ying Hsu', Tsu-Fang Wu', \\ Chen-Han Tsai ${ }^{3}$, Chia-Lin Wu ${ }^{4,5}$, Hui-Yu Wu ${ }^{3}$, Jer-Tsong Hsieh ${ }^{6}$, Chih-Hsin Tang ${ }^{1,7}$ and \\ Chih-Ho Lai ${ }^{1,3,5,8 *}$
}

\begin{abstract}
${ }^{1}$ Graduate Institute of Basic Medical Science, School of Medicine, China Medical University, Taichung, Taiwan, ${ }^{2}$ Department of Applied Cosmetology, Hung Kuang University, Taichung, Taiwan, ${ }^{3}$ Department of Microbiology and Immunology, Graduate Institute of Biomedical Sciences, College of Medicine, Chang Gung University, Taoyuan, Taiwan, ${ }^{4}$ Department of Biochemistry, College of Medicine, Chang Gung University, Taoyuan, Taiwan, ${ }^{5}$ Molecular Infectious Disease Research Center, Department of Neurology, Chang Gung Memorial Hospital, Linkou, Taiwan, ${ }^{6}$ Department of Urology, University of Texas Southwestern Medical Center, Dallas, TX, United States, ${ }^{7}$ Graduate Institute of Biomedical Sciences, School of Medicine, China Medical University, Taichung, Taiwan, ${ }^{8}$ Department of Nursing, Asia University, Taichung, Taiwan
\end{abstract}

OPEN ACCESS

Edited by:

Andres Trostchansky,

Universidad de la República, Uruguay

Reviewed by:

Bilikere S. Dwarakanath,

Shanghai Proton and Heavy lon Center (SPHIC), China

Kanagasabai Vadivel,

David Geffen School of Medicine at UCLA, United States

*Correspondence:

Chih-Ho Lai

chlai@mail.cgu.edu.tw

Specialty section: This article was submitted to Experimental Pharmacology and Drug Discovery,

a section of the journal Frontiers in Pharmacology

Received: 13 February 2018 Accepted: 18 May 2018

Published: 13 June 2018

Citation:

Chen $Y-A$, Shin $H-W$, Lin $Y-C$ Hsu H-Y, Wu T-F, Tsai C-H, Wu C-L,

Wu H-Y, Hsieh J-T, Tang C-H and Lai C-H (2018) Simvastatin Sensitizes Radioresistant Prostate Cancer Cells by Compromising DNA

Double-Strand Break Repair.

Front. Pharmacol. 9:600. doi: 10.3389/fphar.2018.00600
Prostate cancer $(\mathrm{PCa})$ is one of the most prevalent male cancers in western world. Radiation therapy (RT) is commonly used to treat PCa patients. However, a certain proportion of patients develop radioresistant PCa cells, which results in metastatic disease. Statins, which inhibit 3-hydroxy-3-methyl glutaryl coenzyme A (HMG-CoA) reductase, are commonly used to treat hypercholesterolemia, exhibiting beneficial effects on cardiovascular diseases and on several types of cancers, including PCa. However, the mechanistic details and crosstalk between statins and RT in PCa cells remain unknown. In this study, radioresistant DOC-2/DAB2 interactive protein (DAB2IP)deficient PCa cells were used to evaluate whether simvastatin could enhance the effect of ionizing radiation (IR). The crucial molecules that associated with simvastatin elevated radiosensitivity in $\mathrm{PCa}$ cells were explored. Our results demonstrated that a combination treatment with simvastatin and IR synergistically induced apoptosis of radioresistant PCa cells. In addition, simvastatin appeared to compromise DNA double-strand breaks repair by activating the expressions of histone 2A family member $X(\gamma-\mathrm{H} 2 \mathrm{AX})$ and phospho-checkpoint kinase 1 ( $\mathrm{p}-\mathrm{CHK} 1$ ), suggesting an underlying mechanism for this radiosensitization of $\mathrm{PCa}$ cells. These findings reveal that simvastatin may be a potent therapeutic agent for co-treatment with radiation to overcome radioresistance in $\mathrm{PCa}$ cells.

Keywords: cholesterol, HMG-CoA reductase, simvastatin, prostate cancer, radioresistance, DNA double-strand break

\section{INTRODUCTION}

Prostate cancer $(\mathrm{PCa})$ is one of the most prevalent male cancers in western world and the second most frequently diagnosed cancer among males worldwide (Wadajkar et al., 2013). For patients with elevated risk factors or cancer recurrence, radiation therapy (RT) is one of the effective treatment options (Dearnaley et al., 1999; Liu et al., 2014c). However, a significant 
proportion of patients develop metastatic disease where RT is ineffective due to the development of radioresistance in PCa cells (Hummerich et al., 2006; Liu et al., 2014b).

DOC-2/DAB2 interactive protein (DAB2IP), a member of Ras GTPase-activating protein (GAP) family, functions as a tumor suppressor gene to modulate PCa development (Chen et al., 2002; Tsai et al., 2014). Decreased DAB2IP expression enhances PCa cell proliferation and induces epithelial-mesenchymal transition (EMT), resulting in radioresistant ability (Xie et al., 2009, 2010; Kong et al., 2010). Therefore, there is an urgent need to develop new strategies to overcome radioresistance in PCa cells.

Statins are inhibitors of 3-hydroxy-3-methyl-glutaryl coenzyme A (HMG-CoA) reductase that decrease cholesterol synthesis which are necessary molecules for critical cellular functions (Hebert et al., 1997). The effects of statins in enhancing radiosensitivity in several cancer cell types have been reported (Fritz et al., 2003; Lim et al., 2015). Clinical studies have shown that statins decrease the incidence of advanced PCa, including radioresistant PCa (Hindler et al., 2006; Pisanti et al., 2014). In addition, our recent studies using a population-based cohort demonstrated that statin prescriptions remarkably reduced the risk of death in PCa patients undergoing radiotherapy (Sun et al., 2015; Chen et al., 2018). Although the radiosensitizing properties of statins and their potential effects in inhibiting PCa have been reported, the molecular mechanism underlying this outcome has not been clearly explored (Alfaqih et al., 2017).

Several cell-based studies have suggested that statins exert therapeutic effects on PCa cells, but none of these studies employed the DAB2IP knockdown (KD) (shDAB2IP) PC-3 cell line, a more malignant and radioresistant type of PCa cells (Kong et al., 2010; Wu et al., 2013). In this study, we investigated the cytotoxic effects of simvastatin and the molecular mechanisms of its therapeutic effects on radioresistant PCa cells.

\section{MATERIALS AND METHODS}

\section{Antibodies and Reagents}

Antibodies against histone- $\gamma-\mathrm{H} 2 \mathrm{AX}$ and phospho-CHK1 were purchased from Cell Signaling (Danvers, MA, United States). Antibodies specific to PARP, Bcl-2, Bax, cleaved caspase 3, were purchased from Santa Cruz Biotechnology (Santa Cruz, CA, United States). All other chemicals or reagents were purchased from Sigma-Aldrich (St. Louis, MO, United States).

\section{Cell Culture}

The methods for the construction of shDAB2IP and control shVector clones, cell transfection, and clone selection were described previously (Xie et al., 2010). The shRNA system (pGIPZ-lentiviral-shRNAmir from Open Biosystems, Huntsville, $\mathrm{AL}$, United States) was used to $\mathrm{KD}$ endogenous DAB2IP in prostate epithelial cell line, which was selected by using puromycin. The PC3-KD and PC3-Con (control shVector) cell lines were maintained in Roswell Park Memorial Institute (RPMI) 1640 medium (Hyclone, Logan, UT, United States) supplemented with 5\% fetal bovine serum (FBS) (Hyclone). LAPC4-KD and LAPC4-Con (control shVector) cells were maintained in Iscove's modified Dulbecco's medium (IMDM) (Gibco, Grand Island, NY, United States) supplemented with $5 \%$ FBS. The cells were incubated in a humidified atmosphere containing $5 \% \mathrm{CO}_{2}$ at $37^{\circ} \mathrm{C}$.

\section{lonizing Radiation}

Cells were irradiated at room temperature in ambient air using the Faxitron RX-650 irradiator (Faxitron X-ray, Wheeling, IL, United States) at the indicated doses which were needed in each experiment.

\section{Cell Viability Assay}

The sulforhodamine B (SRB) assay (Sigma-Aldrich) was used to determine the effects of simvastatin on the growth of PC3 and LAPC4 cells. Cells were treated with various concentrations of simvastatin for different periods. Cells were fixed and stained with SRB pre-mixed solution $(0.4 \%$ SRB dissolved in $0.1 \%$ acetic acid). Cell viability was determined by a spectrophotometer (BioRad, Hercules, CA, United States) at the wavelength of $565 \mathrm{~nm}$.

\section{Clonogenic Survival Assay}

The clonogenic survival assay was used to analyze the surviving fraction (SF) by following our previous study (Lai et al., 2014). PC3-KD cells were seeded for $24 \mathrm{~h}$ to allow cell attachment and then treated with increasing doses of IR alone (0-6 Gy), or simvastatin $(20 \mu \mathrm{M})$ combined with IR. After 7 days' incubation, the colonies were fixed and stained with $4 \%$ formaldehyde in PBS containing $0.05 \%$ crystal violet. The number of surviving colonies were counted for evaluation of cell viability. Dose-survival curves for each experiment were constructed by plotting the mean SFs semi-logarithmically as a function of IR dose (Chen et al., 2017). The data were analyzed and survival curves were plotted following the linear quadratic (LQ) model using GraphPad Prism 6.0 software (GraphPad Software, United States).

\section{Cell Cycle Analysis}

PC3-KD cells were treated with simvastatin alone $(100 \mu \mathrm{M})$, IR alone (2 Gy), or simvastatin combined with IR for $48 \mathrm{~h}$. The treated cells were prepared and stained with $20 \mu \mathrm{g} / \mathrm{ml}$ propidium iodide (Sigma-Aldrich) for $1 \mathrm{~h}$. The stained cells were determined by FACScalibur flow cytometer (Becton-Dickinson, San Jose, CA, United States) and the cell cycle distribution was assessed by using Cell Quest software WinMDI (Verity Software House, Topsham, ME, United States) as described previously (Lin et al., 2017).

\section{Comet Assay}

Comet assay was employed to determine double-strand breaks (DSBs) in irradiated PC3-KD cells by using a Comet Assay kit (Trevigen, Gaithersburg, MD, United States) according to the manufacturer's instructions. Briefly, cells were mixed with Comet Assay low-melting agarose at a ratio of 1:10 (v/v) and spread evenly on slides. The cells were treated with Comet Assay lysis solution at $4^{\circ} \mathrm{C}$ for $1 \mathrm{~h}$, submerged in cold electrophoresis buffer and subjected to electrophoresis at $21 \mathrm{~V}$ for $30 \mathrm{~min}$. 
The cells were stained with SYBR Gold. The percentage of DNA tail moment were evaluated with the TriTek Comet ScoreTM software (Version 1.5.2.6; TriTek, Corp., Sumerduck, VA, United States).

\section{DNA Ladder Analysis}

The Apoptotic DNA Ladder Detection Kit (Thermo Fisher Scientific, Camarillo, CA, United States) was used to determine the level of DNA fragmentation of apoptotic cells. Briefly, the treated PC3-KD cells $\left(6 \times 10^{5}\right)$ were taken as starting material for DNA isolation. Cells were washed with PBS and centrifuged at 1,200 rpm before lysis of the cell pellet. Cells were incubated with Enzyme A at $37^{\circ} \mathrm{C}$ for $10 \mathrm{~min}$ and added Enzyme $\mathrm{B}$ then incubated at $50^{\circ} \mathrm{C}$ for 30 min. DNA was isolated by absolute ethanol and then dissolved in DNA suspension buffer. DNA solution was loaded on $1.2 \%$ agarose gel for electrophoresis. Ethidium bromide-stained DNA was visualized under UV light and analyzed.

\section{Western Blot Analysis}

PC3-KD cells were treated with simvastatin alone $(100 \mu \mathrm{M})$, IR alone (2 Gy), or simvastatin combined with IR for $48 \mathrm{~h}$. The treated cells were harvested and cell lysates were prepared. The samples were then resolved by $10 \%$ SDS-PAGE and transferred onto polyvinylidene difluoride (PVDF) membranes (Millipore, Billerica, MA, United States). Membranes were probed with primary antibodies and then incubated with horseradish peroxidase (HRP)-conjugated secondary antibody. The proteins of interest were detected using the ECL Western Blotting Detection Reagents (RE Healthcare, Little Chalfont, United Kingdom) and visualized using Image Quant LAS4000 and TL software (GE Healthcare). The signal intensity of each protein was quantified with the UN-SCAN-IT software (Silk Scientific Corporation, Orem, UT, United States).

\section{Immunofluorescence Staining}

PC3-KD cells $\left(2 \times 10^{5}\right.$ cells/well $)$ were seeded on $13-\mathrm{mm}$ glass coverslip in 6-well plates. After treatment, cells were washed by PBS and fixed with $1 \%$ paraformaldehyde (Sigma-Aldrich) and permeabilized with $0.1 \%$ Triton $\mathrm{X}-100$ for $10 \mathrm{~min}$. The samples were incubated with $\gamma$-H2AX antibody. Samples were washed and then incubated with Alexa Fluor 488 conjugated anti-rabbit antibody (Invitrogen, Carlsbad, CA, United States) for $1 \mathrm{~h}$. Nuclei were counterstained with 4',6-diamidino-2phenylindole (DAPI) $(0.2 \mu \mathrm{g} / \mathrm{ml})$ for $15 \mathrm{~min}$. The stained cells were then analyzed under a fluorescence microscope (Carl Zeiss, Göttingen, Germany) with a $63 \times$ objective (oil immersion, aperture 1.3). All samples were examined in three independent experiments.

\section{Statistical Analysis}

Statistics analysis comparisons of two groups were evaluated by using Student's $t$-test and showed as mean \pm standard deviation. $P<0.05$ was considered statistically significant. The statistical software was the SPSS program (version 18.0 for windows, SPSS, Inc., Chicago, IL, United States).

\section{RESULTS}

\section{Simvastatin Inhibited PCa Cell Proliferation}

To determine the therapeutic potential of statins, we first analyzed whether simvastatin had a cytotoxic effect on radioresistant $\mathrm{PCa}$ cells using a DAB2IP $\mathrm{KD}$ radioresistant cell lines (Kong et al., 2010). Two DAB2IP-KD clones, PC3-KD and LAPC4-KD, were investigated in this study. Western blot analysis showed that DAB2IP levels were significantly reduced in both DAB2IP-KD lines compared to those in each DAB2IP-control (shVector) cell line (Supplementary Figure S1A). In addition, our data showed that simvastatin effectively inhibited cell proliferation in both LAPC4-KD and PC3-KD lines (Supplementary Figure S1B). Since simvastatin induced considerably higher apoptosis in PC3KD cells than that in LAPC4-KD cells, we therefore chose PC3-KD cells as an assay platform for the subsequent experiments. In addition, PZ-HPV-7 cells, an immortalized line derived from benign prostate cells showed sensitizing to radiation (Kong et al., 2015), were used as control group. Cells were cultured with increasing concentrations of simvastatin $(0-500 \mu \mathrm{M})$ for $48 \mathrm{~h}$, and cell viability was analyzed by SRB assay. Our results indicated that simvastatin hardly showed any effect on the viability of PZ-HPV-7 cells (Figure 1A). However, PC3-KD cell proliferation was effectively inhibited by treatment with simvastatin in a concentrationand time-dependent manners (Figures 1A,B) with an approximate half-maximal inhibitory concentration $\left(\mathrm{IC}_{50}\right)$ of $100 \mu \mathrm{M}$.

Due to the cytotoxic activity of simvastatin on radioresistant PCa cells, we next determined whether simvastatin sensitized $\mathrm{PCa}$ cells to radiation. PC3-KD cells were treated with simvastatin alone $(100 \mu \mathrm{M})$, ionizing radiation (IR) alone (2 Gy), or a combination of simvastatin and IR. Our data showed that survival of PC3-KD cells was significantly decreased after treatment with simvastatin alone or simvastatin plus IR (Figure 1C). These results suggest that simvastatin exhibits a significant cytotoxic effect against radioresistant $\mathrm{PCa}$ cells.

\section{Simvastatin Synergistically Sensitized Radioresistant PCa Cells to Radiation}

We then examined whether simvastatin could increase susceptibility of radioresistant PCa cells to radiation. PC3KD cells were treated with IR alone (0-6 Gy) or simvastatin (20 $\mu \mathrm{M})$ combined with IR, and survival was assessed using a clonogenic survival assay. As shown in Figure 2, simvastatin effectively enhanced the radiosensitivity of PC3-KD cells with increasing doses of IR. In addition, the combined treatment with simvastatin and IR significantly increased the susceptibility of cells to IR compared to 
A

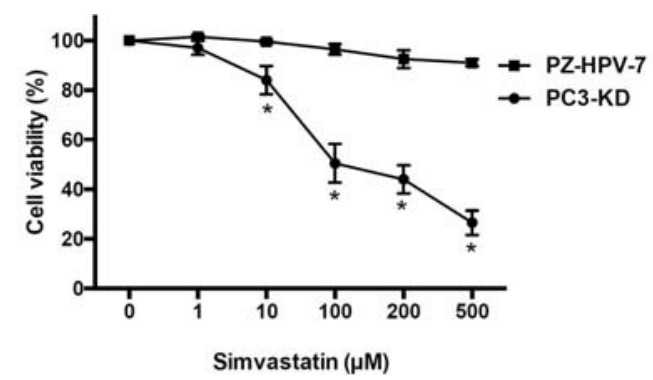

C

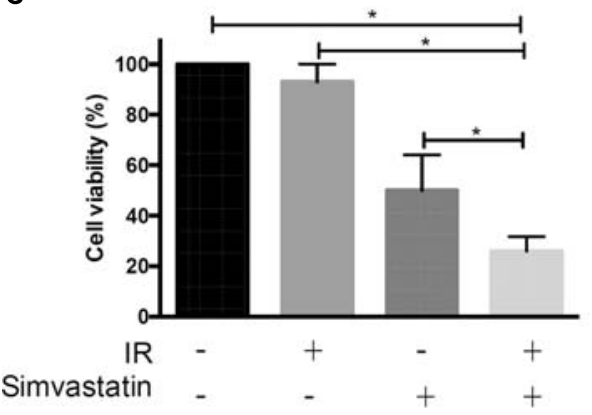

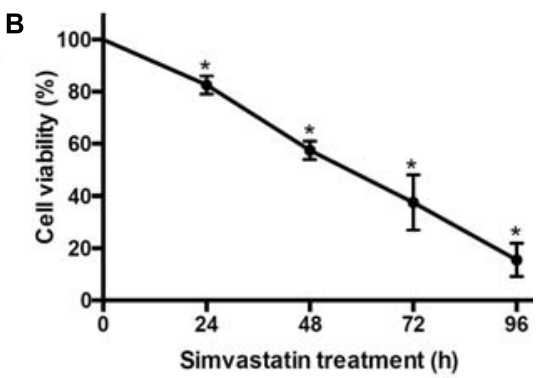

FIGURE 1 | Simvastatin inhibited radioresistant PCa cell proliferation. Cell viability of PZ-HPV-7 and PC3-KD cells in response to simvastatin treatment at various concentrations and durations: (A) Simvastatin at various concentrations ranging from 0 to $500 \mu \mathrm{M}$ was added to cells for $48 \mathrm{~h}$. (B) PC3-KD cells were exposed to $100 \mu \mathrm{M}$ simvastatin for the times indicated $(0,24,48,72$, and $96 \mathrm{~h})$. (C) Cells were treated with ionizing radiation (IR) alone (2 Gy), simvastatin alone (100 $\mu \mathrm{M})$, or simvastatin combined with IR and were compared to untreated cells. The cell viability was analyzed by sulforhodamine B (SRB) assay. The asterisk $(*)$ indicates statistical significance $(P<0.05)$ as determined by Student's $t$-test.

treatment with IR alone. These results demonstrate that simvastatin sensitizes radioresistant $\mathrm{PCa}$ cells to radiation.

\section{Simvastatin Induced Apoptosis in Radioresistant PCa Cells}

To further assess whether simvastatin induced apoptosis in PCa cells, PC3-KD cells were treated with simvastatin alone $(100 \mu \mathrm{M})$, IR alone (2 Gy), or a combination of simvastatin and IR for 0 , 12,24 , and $48 \mathrm{~h}$. The distributions of cells in each cell cycle state were determined by flow cytometry. As shown in Figure 3, a low percentage of mock and IR-treated cells were in the sub-G1 state from 0 to $48 \mathrm{~h}$. After treating simvastatin for $48 \mathrm{~h}$, the sub-G1 cell population $(9.30 \%)$ was significantly increased compared to the mock group (1.03\%). Notably, there was a dramatic increase in sub-G1 population upon co-treatment with IR and simvastatin following incubation for $48 \mathrm{~h}(21.56 \%)$. These results indicate that simvastatin and IR synergistically enhance cell death in radioresistant $\mathrm{PCa}$ cells through apoptosis.

\section{Co-treatment With Simvastatin and Radiation Increased Biomarkers of DNA Double-Strand Breaks and Apoptosis in Radiosensitive PCa Cells}

To investigate the molecular mechanism underlying the enhanced IR-induced cell death by simvastatin in PC3-KD cells, the expression levels of key molecules associated with radiation-induced DNA DSBs were investigated. As shown in
Figure 4A, the expressions of histone 2A family member $\mathrm{X}$ $(\gamma-\mathrm{H} 2 \mathrm{AX})$ and phospho-checkpoint kinase 1 (p-CHK1) were slightly increased in cells treated with IR alone compared to the mock or simvastatin-treated groups. However, the expression levels of these phospho-proteins were remarkably increased following co-treatment with simvastatin and IR. We then analyzed the levels of molecules involved in apoptosis. As shown in Figure 4B, there were significant increases in activated PARP and cleaved-caspase 3 in cells co-treated with simvastatin and IR. In addition, simvastatin plus IR treatment effectively inhibited the expression of the anti-apoptotic molecule, Bcl-2, and stimulated the expression of the apoptosis-promoting proteins, Bax and Bak. We then performed DNA ladder assay to examine cell apoptosis. As shown in Supplementary Figure S2, mock treatment and IR did not present significant fragmentation. In contrast, cells treated with simvastatin plus IR and incubated for $48 \mathrm{~h}$ showed laddering of DNA, which is a characteristic feature of cell apoptosis. These results suggest that simvastatin increases radiation-induced DSB and apoptosis in radioresistant PCa cells.

\section{Simvastatin-Enhanced Radiosensitivity Was Associated With Compromised DSB Repair}

Recruitment of $\gamma-\mathrm{H} 2 \mathrm{AX}$ and 53BP1 at sites of DNA damage in the nucleus is an early response to DSB (Kong et al., 2010). We therefore examined simvastatin-induced cell death in PCa cells by analyzing foci formation of $\gamma$-H2AX and 53BP1. PC3-KD cells were treated with IR (2 Gy) or simvastatin 

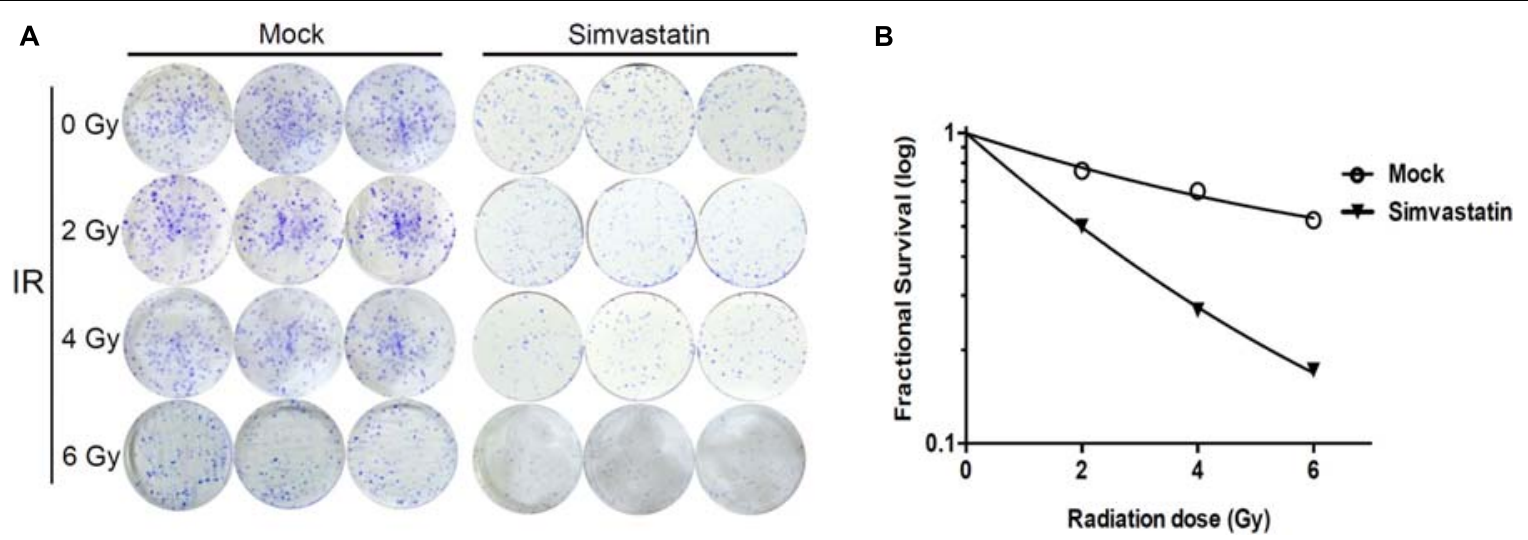

FIGURE 2 | Combined treatment with simvastatin and IR effectively induced cell death. PC3-KD cells were treated with IR alone (0-6 Gy) or simvastatin (20 $\mu$ M) combined with IR. After incubating for 7 days, cells were (A) stained with crystal violet and (B) assessed for survival using clonogenic assay as described in Section "Materials and Methods."

A

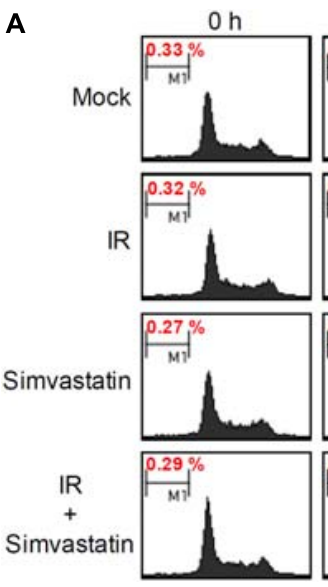

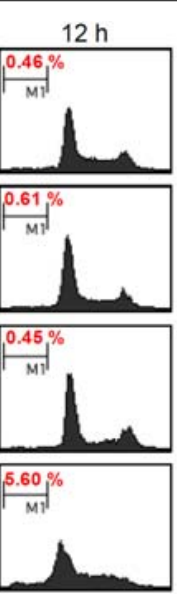

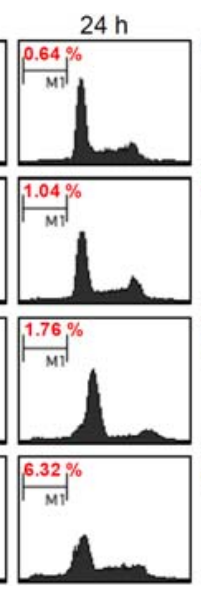

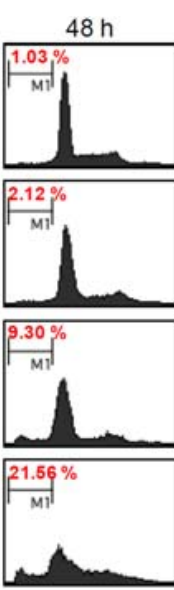

B

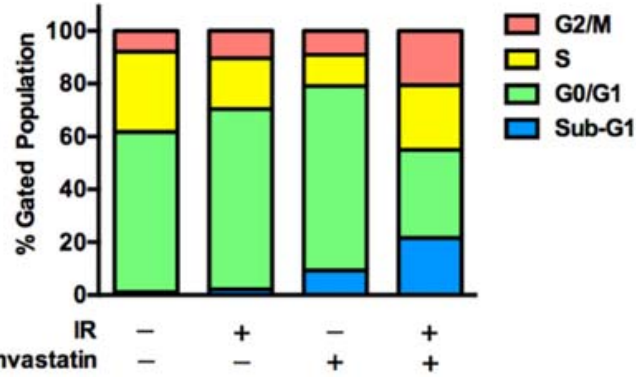

FIGURE 3 | Simvastatin induced apoptosis in radioresistant PCa cells. (A) PC3-KD cells were untreated (control), exposed to IR alone (2 Gy), simvastatin alone $(100 \mu \mathrm{M})$, or simvastatin plus IR, and incubated for $0,12,24$, and $48 \mathrm{~h}$. Cell cycle distributions based on DNA content was analyzed using flow cytometry.

(B) The percentages of cells in each phase were calculated.

(100 $\mu \mathrm{M}$ ) plus IR between 0 and $48 \mathrm{~h}$, and subjected to immunofluorescence staining to detect $\gamma$-H2AX and 53BP1 foci. As shown in Figure 5, nuclear co-localization of $\gamma-\mathrm{H} 2 \mathrm{AX}$ (red) and 53BP1 (green) foci significantly increased in PC3$\mathrm{KD}$ cells when exposed to IR (2 Gy) for $0.5 \mathrm{~h}$ compared to the untreated group $(0 \mathrm{~h})$. The foci formation was observed for $48 \mathrm{~h}$ in cells co-treated with simvastatin and IR. However, decreased foci levels were observed in PC3-KD cells treated with IR alone for $48 \mathrm{~h}$ compared to those co-treated with simvastatin and IR for $48 \mathrm{~h}$. To strengthen our findings that higher levels of $\gamma-\mathrm{H} 2 \mathrm{AX}$ at 24 and $48 \mathrm{~h}$ after irradiation shows persistence of DSB, we conducted comet assays. As shown in Figure 6, the comets were significantly increased in cells treated with a combination of IR and statin compared with IR treatment alone at 24 and $48 \mathrm{~h}$. Following $48 \mathrm{~h}$ incubation, IR only slightly increased the comets. Nevertheless, in cells treated with IR plus simvastatin, high levels of comets remained observable at $48 \mathrm{~h}$. Together, these results demonstrate that simvastatin sustains IR-induced DSB in radioresistant PCa cells.

\section{DISCUSSION}

Radiation therapy is used to damage DNA in PCa cells by IR to increase apoptosis (Chang et al., 2014). However, a certain proportion of patients do not respond to this treatment and become metastatic disease due to intrinsic or the development of radioresistance in PCa cells (Hummerich et al., 2006; Liu et al., 2014a). In this study, we have shown that the co-treatment of simvastatin and IR enhanced susceptibility of PCa cells to IR compared to IR treatment alone. These results demonstrate that simvastatin and IR work synergistically to enhance radiationinduced cell death in radioresistant PCa cells.

Statins that inhibit HMG-CoA reductase, the rate-limiting enzyme in cholesterol biosynthesis, are widely prescribed for 

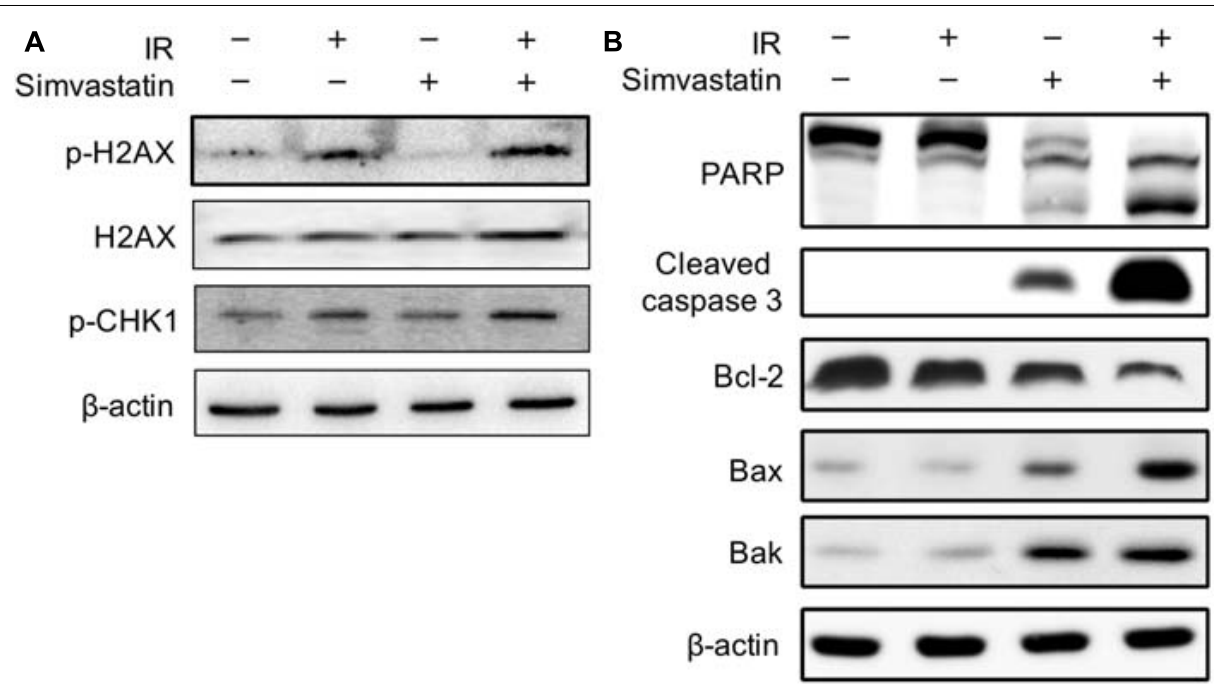

FIGURE 4 | Effects of simvastatin on DSB- and apoptosis-related molecules. PC3-KD cells were treated with IR alone (2 Gy), simvastatin alone (100 $\mu$ M) or a combination of simvastatin and IR, then incubated for $48 \mathrm{~h}$. Cell lysates were prepared and subjected to western blot analysis using antibodies to probe for the key molecules of (A) DSB and (B) apoptosis. The data shown are a representative of three independent experiments. $\beta$-actin was used as a loading control.
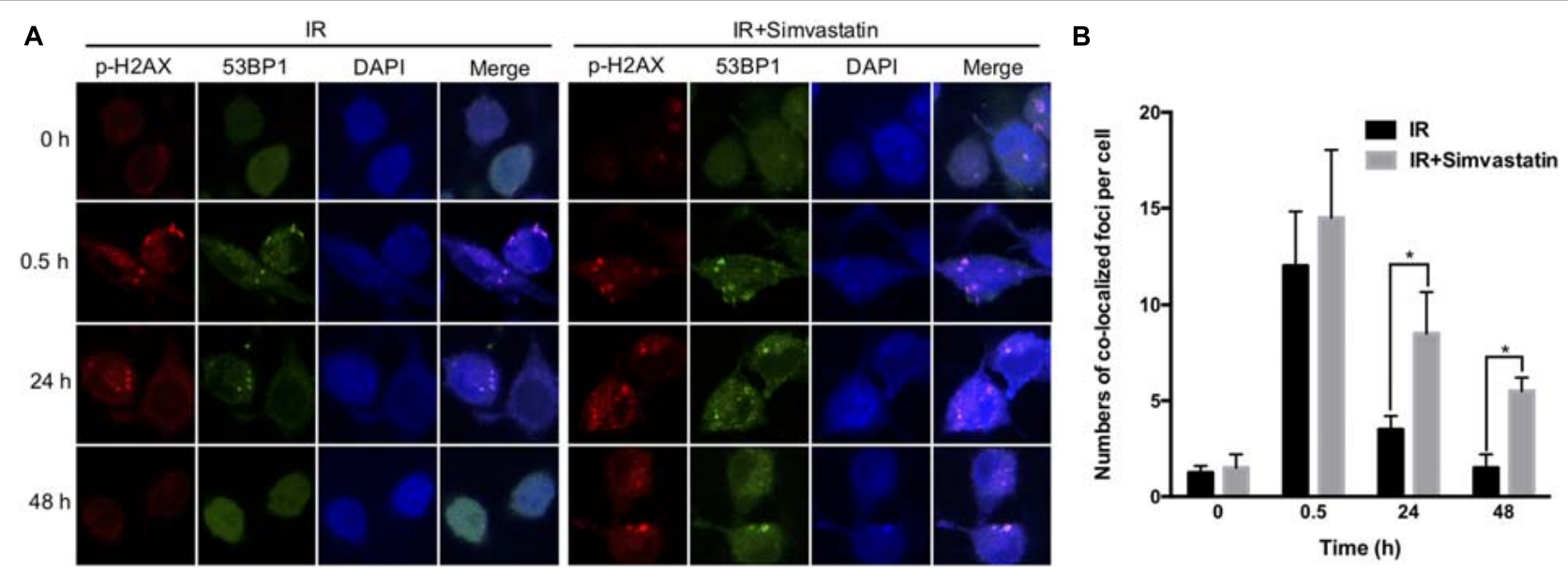

FIGURE 5 | Simvastatin sensitized PCa cells to radiation by compromising DSB repair. PC3-KD cells were treated to IR alone (2 Gy) or co-treated with simvastatin $(100 \mu \mathrm{M})$ and IR for the indicated times (0, 0.5, 24, and 48 h). (A) Cells were immunostained with $\gamma$-H2AX (red), 53BP1 (green) and DAPI (blue).

(B) The colocalization of $\gamma$-H2AX with 53BP1 foci in the nuclei were counted for each time point. Statistical significance was evaluated using Student's $t$-test $\left({ }^{*} P<0.05\right)$.

lowering serum cholesterol (Hebert et al., 1997). Several preclinical studies indicated that statins prevent proliferation of PCa cells by interfering with these pathways in various ways. In a bone marrow stroma (BMS) model, Brown et al. (2012) showed that statins dramatically reduced prostate epithelial cell invasion toward BMS. The authors suggested that geranylgeranyl pyrophosphate and mevalonate, precursors in cholesterol synthesis, restored the invasive ability of PCa cells treated with simvastatin (Brown et al., 2012). In addition, statins impair the tumor metastasis by inhibiting extracellular matrix degradation, cell migration, and invasion (Hindler et al., 2006). The protective function of stains, included reducing risk of $\mathrm{PCa}$ cells that arrest cell cycle, induce apoptosis, leading to autophagy and inhibition of tumor metastasis (Papadopoulos et al., 2011; Zhang et al., 2013). Hoque et al. (2008) reported that apoptosis of PCa cells was induced by lovastatin and simvastatin through both cytochrome $c$-dependent and -independent signaling pathways, involving caspase 3, 8, and 9. Statins may sensitize cells to radiation by arresting cell cycle in the late G1 phase (Chan et al., 2003). Further, our current study focused on the molecular mechanism of statin effects, which effectively enhanced radiation-induced DSB to cause apoptosis. Taken together, these results suggest that statins act as a radiosensitizer and may be used as new therapeutic strategy to treat PCa in combination with RT. 


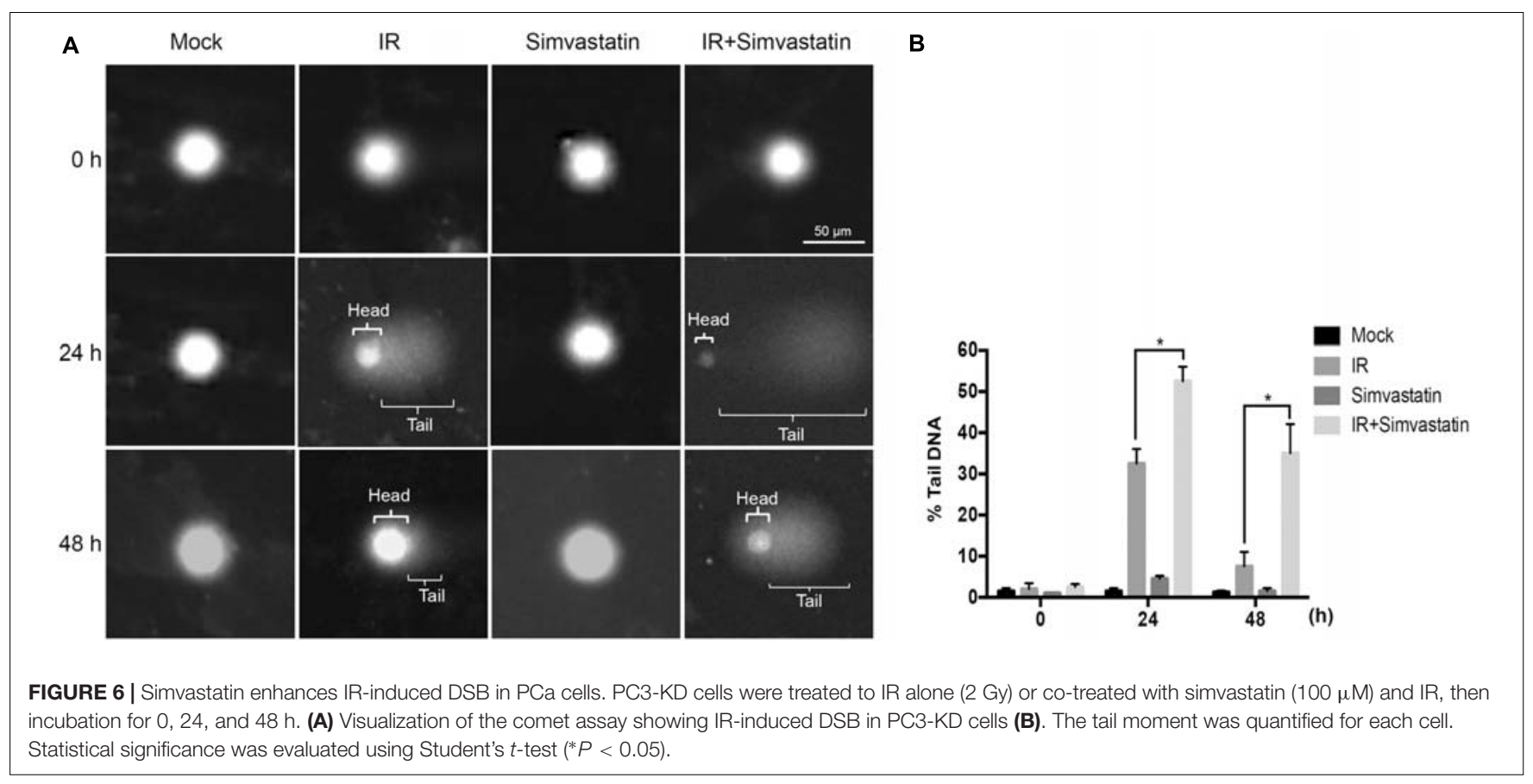

Increased DSB are accompanied by the formation of histone $\gamma$-H2AX molecules in the cell nuclei (Bonner et al., 2008). The triggering of checkpoint responses to radiation is one of the major barriers for preventing carcinogenesis (Bartek et al., 2007). In this study, co-treatment with simvastatin and IR highly elevated the expressions of phospho-proteins, including $\gamma-\mathrm{H} 2 \mathrm{AX}$ and $\mathrm{p}-\mathrm{CHK} 1$. In addition, simvastatin promoted radiation-induced cell death in PCa cells and increased $\gamma-\mathrm{H} 2 \mathrm{AX}$ foci-formation associated with DSB. Our results reveal that the mechanisms underlying radiosensitization induced by simvastatin in PCa cells involves triggering the $\mathrm{CHK} 1$ checkpoint response and promotes DSB.

It has been shown that statin was able to counteract radiationstimulated lung cancer extravasation and metastasis in mice (Hamalukic et al., 2011). The mechanism of statin enhanced radiosensitivity in lung cancer was mediated by inhibiting the PI3K/AKT and activating the tumor suppressor AMP-activated kinase (AMPK) pathway (Sanli et al., 2011). Similar results were obtained by using xenograft mouse models showing that statin sensitized gastric and colorectal cancer cells to radiotherapy through modulation of the ATM/AMPK/p21 pathway (Lim et al., 2015). Our present study showed that the combination therapy using simvastatin and radiation significantly elevated the expression of $\gamma$-H2AX and $\mathrm{p}$-CHK1, which enhanced the occurrence of DSB; these observations may be due to the activation of ATM.

A recent study showed conflicting results indicated that lovastatin mediates protection from radiation-induced lung injury (Ziegler et al., 2017). This protection of lung endothelial cells from radiation-induced caspase-dependent apoptosis may involve p53-regulated mechanisms. In contrast, it showed that statin enhanced sensitivity to radiotherapy in lung cancer by inhibiting the p53/p21 signaling pathway (Sanli et al.,
2011). A similar study reported that simvastatin synergistically enhanced the effects of radiation in gastric and colorectal cancer cells (Lim et al., 2015). Our present study showed that simvastatin enhanced radiation-induced apoptosis by inhibiting DNA repair in PC3 cells, which were p53-deficient. Therefore, this may explain the cell type-specific diverse effects of statin on IRinduced apoptosis and DSB repair (Ziegler et al., 2017).

Although our study has demonstrated that simvastatin functions as a radiosensitizer and may be used to treat PCa in combination with RT, the limitation of this study is that it lacks in vivo data. Accordingly, it is worth to investigate in vivo, which will fill the gap in the translational studies.

\section{CONCLUSION}

Our findings demonstrate that simvastatin can enhance the effects of IR in radioresistant PCa cells. The molecular mechanism of this radiosensitization may be attributed to enhancement of the checkpoint response process and sustained reduction of the DSB repair pathway. This study indicates that simvastatin could be a potential personalized medicine in combination with radiotherapy to target PCa cells, particularly those that are radioresistant.

\section{AUTHOR CONTRIBUTIONS}

J-TH, C-HTang, and C-HL: conception or design of this work. Y-AC, H-WS, Y-CL, H-YH, C-LW, and C-HTsai: experimental study. Y-AC, H-WS, Y-CL, H-YH, and C-HTsai: data analysis and interpretation. Y-AC, C-HTang, and C-HL: writing the manuscript. All authors provided final approval of the manuscript. 


\section{FUNDING}

This work was supported by the Ministry of Science and Technology (105-2313-B-182-001 and 106-2320-B-182-012MY3), the Chang Gung Memorial Hospital (CMRPD1F0011-3, CMRPD1F0431-3, and BMRPE90), and the Tomorrow Medical Foundation.

\section{ACKNOWLEDGMENTS}

The authors would like to thank the editor and the reviewers for the editorial assistance and their valuable comments. They also sincerely appreciate the assistance of Shu-Chen Shen for analyzing confocal microscopy at the Division of Instrument Service of Academia Sinica, Taipei, Taiwan; Microscopy Center at Chang Gung University; and Ya-Fen Lu for analyzing flow cytometry at the Core

\section{REFERENCES}

Alfaqih, M. A., Allott, E. H., Hamilton, R. J., Freeman, M. R., and Freedland, S. J. (2017). The current evidence on statin use and prostate cancer prevention: are we there yet? Nat. Rev. Urol. 14, 107-119. doi: 10.1038/nrurol.2016.199

Bartek, J., Bartkova, J., and Lukas, J. (2007). DNA damage signalling guards against activated oncogenes and tumour progression. Oncogene 26, 7773-7779. doi: 10.1038/sj.onc.1210881

Bonner, W. M., Redon, C. E., Dickey, J. S., Nakamura, A. J., Sedelnikova, O. A., Solier, S., et al. (2008). GammaH2AX and cancer. Nat. Rev. Cancer 8, 957-967. doi: $10.1038 / \mathrm{nrc} 2523$

Brown, M., Hart, C., Tawadros, T., Ramani, V., Sangar, V., Lau, M., et al. (2012). The differential effects of statins on the metastatic behaviour of prostate cancer. Br. J. Cancer 106, 1689-1696. doi: 10.1038/bjc.2012.138

Chan, K. K., Oza, A. M., and Siu, L. L. (2003). The statins as anticancer agents. Clin. Cancer Res. 9, 10-19.

Chang, L., Graham, P. H., Hao, J., Ni, J., Bucci, J., Cozzi, P. J., et al. (2014). PI3K/Akt/mTOR pathway inhibitors enhance radiosensitivity in radioresistant prostate cancer cells through inducing apoptosis, reducing autophagy, suppressing NHEJ and HR repair pathways. Cell Death Dis. 5:e1437. doi: $10.1038 /$ cddis.2014.415

Chen, H., Pong, R. C., Wang, Z., and Hsieh, J. T. (2002). Differential regulation of the human gene DAB2IP in normal and malignant prostatic epithelia: cloning and characterization. Genomics 79, 573-581. doi: 10.1006/geno.2002.6739

Chen, Y. A., Lien, H. M., Kao, M. C., Lo, U. G., Lin, L. C., Lin, C. J., et al. (2017). Sensitization of radioresistant prostate cancer cells by resveratrol isolated from arachis hypogaea stems. PLoS One 12:e0169204. doi: 10.1371/journal.pone. 0169204

Chen, Y. A., Lin, Y. J., Lin, C. L., Lin, H. J., Wu, H. S., Hsu, H. Y., et al. (2018). Simvastatin therapy for drug repositioning to reduce the risk of prostate cancer mortality in patients with hyperlipidemia. Front. Pharmacol. 9:225. doi: 10.3389/fphar.2018.00225

Dearnaley, D. P., Khoo, V. S., Norman, A. R., Meyer, L., Nahum, A., Tait, D., et al. (1999). Comparison of radiation side-effects of conformal and conventional radiotherapy in prostate cancer: a randomised trial. Lancet 353, 267-272. doi: 10.1016/S0140-6736(98)05180-0

Fritz, G., Brachetti, C., and Kaina, B. (2003). Lovastatin causes sensitization of HeLa cells to ionizing radiation-induced apoptosis by the abrogation of G2 blockage. Int. J. Radiat. Biol. 79, 601-610. doi: 10.1080/09553000310001609233

Hamalukic, M., Huelsenbeck, J., Schad, A., Wirtz, S., Kaina, B., and Fritz, G. (2011). Rac1-regulated endothelial radiation response stimulates extravasation and metastasis that can be blocked by HMG-CoA reductase inhibitors. PLoS One 6:e26413. doi: 10.1371/journal.pone.0026413

Hebert, P. R., Gaziano, J., Chan, K., and Hennekens, C. H. (1997). Cholesterol lowering with statin drugs, risk of stroke, and total
Instrument Center, Chang Gung University, Taoyuan, Taiwan.

\section{SUPPLEMENTARY MATERIAL}

The Supplementary Material for this article can be found online at: https://www.frontiersin.org/articles/10.3389/fphar. 2018.00600/full\#supplementary-material

FIGURE S1 | Simvastatin increased radio-sensitivity in DAB2IP-knockdown PCa cells. (A) Western blot analysis of DAB2IP expression in DAB2IP-control (shVector) and knockdown (shDAB2IP) PCa cells. (B) Cell viability of DAB2IP-knockdown cells in response to simvastatin and/or IR was analyzed. Statistical significance was evaluated using Student's t-test $\left({ }^{*} P<0.05\right)$.

FIGURE S2 | Analysis of DNA ladder in apoptotic PC3-KD cells. PC3-KD cells were treated to ionizing radiation (IR) alone (2 Gy) or co-treated with simvastatin $(100 \mathrm{M})$ and IR, then incubation for $48 \mathrm{~h}$. DNA was extracted from cells and subjected to agarose gel electrophoresis. M, $1 \mathrm{~Kb}$ ladder.

mortality: an overview of randomized trials. JAMA 278, 313-321. doi: 10.1001/jama.1997.03550040069040

Hindler, K., Cleeland, C. S., Rivera, E., and Collard, C. D. (2006). The role of statins in cancer therapy. Oncologist 11, 306-315. doi: 10.1634/theoncologist.11-3-306 Hoque, A., Chen, H., and Xu, X. C. (2008). Statin induces apoptosis and cell growth arrest in prostate cancer cells. Cancer Epidemiol. Biomarkers Prev. 17, 88-94. doi: 10.1158/1055-9965.EPI-07-0531

Hummerich, J., Werle-Schneider, G., Popanda, O., Celebi, O., Chang-Claude, J., Kropp, S., et al. (2006). Constitutive mRNA expression of DNA repairrelated genes as a biomarker for clinical radio-resistance: a pilot study in prostate cancer patients receiving radiotherapy. Int. J. Radiat. Biol. 82, 593-604. doi: 10.1080/09553000600883302

Kong, D., Sethi, S., Li, Y., Chen, W., Sakr, W. A., Heath, E., et al. (2015). Androgen receptor splice variants contribute to prostate cancer aggressiveness through induction of EMT and expression of stem cell marker genes. Prostate 75, 161-174. doi: 10.1002/pros.22901

Kong, Z., Xie, D., Boike, T., Raghavan, P., Burma, S., Chen, D. J., et al. (2010). Downregulation of human DAB2IP gene expression in prostate cancer cells results in resistance to ionizing radiation. Cancer Res. 70, 2829-2839. doi: 10.1158/0008-5472.CAN-09-2919

Lai, C. H., Chang, C. S., Liu, H. H., Tsai, Y. S., Hsu, F. M., Yu, Y. L., et al. (2014). Sensitization of radio-resistant prostate cancer cells with a unique cytolethal distending toxin. Oncotarget 5, 5523-5534. doi: 10.18632/oncotarget. 2133

Lim, T., Lee, I., Kim, J., and Kang, W. K. (2015). Synergistic effect of simvastatin plus radiation in gastric cancer and colorectal cancer: implications of birc 5 and connective tissue growth factor. Int. J. Radiat. Oncol. Biol. Phys. 93, 316-325. doi: 10.1016/j.ijrobp.2015.05.023

Lin, H. J., Liu, H. H., Lin, C. D., Kao, M. C., Chen, Y. A., Chiang-Ni, C., et al. (2017). Cytolethal distending toxin enhances radiosensitivity in prostate cancer cells by regulating autophagy. Front. Cell Infect. Microbiol. 7:223. doi: 10.3389/ fcimb.2017.00223

Liu, H.-H., Tsai, Y.-S., Lai, C.-L., Tang, C.-H., Lai, C.-H., Wu, H.-C., et al. (2014a). Evolving personalized therapy for castration-resistant prostate cancer. Biomedicine (Taipei) 4, 1-9 doi: 10.7603/s40681-0140002-5

Liu, H. H., Tsai, Y. S., Lai, C. L., Tang, C. H., Lai, C. H., Wu, H. C., et al. (2014b). Evolving personalized therapy for castration-resistant prostate cancer. Biomedicine (Taipei) 4:2. doi: 10.7603/s40681-014-0002-5

Liu, H. H., Tsai, Y. S., Lai, C. L., Tang, C. H., Lai, C. H., Wu, H. C., et al. (2014c). Evolving personalized therapy for castration-resistant prostate cancer. Biomedicine (Taipei) 4, 7-15. doi: 10.7603/s40681-014-0002-5

Papadopoulos, G., Delakas, D., Nakopoulou, L., and Kassimatis, T. (2011). Statins and prostate cancer: molecular and clinical aspects. Eur. J. Cancer 47, 819-830. doi: 10.1016/j.ejca.2011.01.005 
Pisanti, S., Picardi, P., Ciaglia, E., D’Alessandro, A., and Bifulco, M. (2014). Novel prospects of statins as therapeutic agents in cancer. Pharmacol. Res. 88, 84-98. doi: 10.1016/j.phrs.2014.06.013

Sanli, T., Liu, C., Rashid, A., Hopmans, S. N., Tsiani, E., Schultz, C., et al. (2011). Lovastatin sensitizes lung cancer cells to ionizing radiation: modulation of molecular pathways of radioresistance and tumor suppression. J. Thorac. Oncol. 6, 439-450. doi: 10.1097/JTO.0b013e3182 $049 \mathrm{~d} 8 \mathrm{~b}$

Sun, L. M., Lin, M. C., Lin, C. L., Chang, S. N., Liang, J. A., Lin, I. C., et al. (2015). Statin use reduces prostate cancer all-cause mortality: a nationwide population-based cohort study. Medicine (Baltimore) 94:e1644. doi: 10.1097/ MD.0000000000001644

Tsai, Y. S., Lai, C. L., Lai, C. H., Chang, K. H., Wu, K., Tseng, S. F., et al. (2014). The role of homeostatic regulation between tumor suppressor DAB2IP and oncogenic Skp2 in prostate cancer growth. Oncotarget 5, 6425-6436. doi: 10.18632 /oncotarget. 2228

Wadajkar, A. S., Menon, J. U., Tsai, Y. S., Gore, C., Dobin, T., Gandee, L., et al. (2013). Prostate cancer-specific thermo-responsive polymer-coated iron oxide nanoparticles. Biomaterials 34, 3618-3625. doi: 10.1016/j.biomaterials.2013. 01.062

Wu, K., Xie, D., Zou, Y., Zhang, T., Pong, R. C., Xiao, G., et al. (2013). The mechanism of DAB2IP in chemoresistance of prostate cancer cells. Clin. Cancer Res. 19, 4740-4749. doi: 10.1158/1078-0432.CCR-13-0954

Xie, D., Gore, C., Liu, J., Pong, R. C., Mason, R., Hao, G., et al. (2010). Role of DAB2IP in modulating epithelial-to-mesenchymal transition and prostate cancer metastasis. Proc. Natl. Acad. Sci. U.S.A. 107, 2485-2490. doi: 10.1073/ pnas.0908133107

Xie, D., Gore, C., Zhou, J., Pong, R. C., Zhang, H., Yu, L., et al. (2009). DAB2IP coordinates both PI3K-Akt and ASK1 pathways for cell survival and apoptosis. Proc. Natl. Acad. Sci. U.S.A. 106, 19878-19883. doi: 10.1073/pnas.090845 8106

Zhang, J., Yang, Z., Xie, L., Xu, L., Xu, D., and Liu, X. (2013). Statins, autophagy and cancer metastasis. Int. J. Biochem. Cell Biol. 45, 745-752. doi: 10.1016/j.biocel. 2012.11.001

Ziegler, V., Henninger, C., Simiantonakis, I., Buchholzer, M., Ahmadian, M. R., Budach, W., et al. (2017). Rho inhibition by lovastatin affects apoptosis and DSB repair of primary human lung cells in vitro and lung tissue in vivo following fractionated irradiation. Cell Death Dis. 8:e2978. doi: 10.1038/cddis.2017.372

Conflict of Interest Statement: The authors declare that the research was conducted in the absence of any commercial or financial relationships that could be construed as a potential conflict of interest.

Copyright (c) 2018 Chen, Shih, Lin, Hsu, Wu, Tsai, Wu, Wu, Hsieh, Tang and Lai. This is an open-access article distributed under the terms of the Creative Commons Attribution License (CC BY). The use, distribution or reproduction in other forums is permitted, provided the original author(s) and the copyright owner are credited and that the original publication in this journal is cited, in accordance with accepted academic practice. No use, distribution or reproduction is permitted which does not comply with these terms. 